\title{
Density functional theory within spin-orbit coupling and hubbard correction for investigation of optoelectronic properties of the orthorhombic perovskite $\mathrm{LaPdO}_{3}$
}

\author{
Souraya Goumri-Said ${ }^{\text {a, * }}$, Sikander Azam ${ }^{\text {b }}$, Saleem Ayaz Khan ${ }^{\text {c, }}$ \\ Mohammed Benali Kanoun ${ }^{\mathrm{d}}$ \\ ${ }^{a}$ College of Science, Physics Department, Alfaisal University, P.O. Box 50927, Riyadh, 11533, Saudi Arabia \\ ${ }^{\mathrm{b}}$ Faculty of Engineering and Applied Sciences, Department of Physics, RIPHAH International University I-14 Campus Islamabad, Pakistan \\ ${ }^{\mathrm{c}}$ New Technologies - Research Center, University of West Bohemia, Univerzitni 8, 306 14, Pilsen, Czech Republic \\ d Physics Department, College of Science, King Faisal University, P.O. Box 400, Al-Ahsa, 31982, Saudi Arabia
}

\section{A R T I C L E I N F O}

\section{Article history:}

Received 17 March 2019

Received in revised form

30 April 2019

Accepted 30 April 2019

\section{Keywords:}

$\mathrm{LaPdO}_{3}$

Perovskites

Electronic structure

Elastic properties

Optical properties

DFT

\begin{abstract}
A B S T R A C T
To apprehend the electronic structures and related physical quantities such as Fermi surface, and optical properties of the perovskite $\mathrm{LaPdO}_{3}$ system, we employed the full potential linearized augmented plane wave method. The existence of rare-earth (La) and the actinide element (Pd) has implied to include the Hubbard correction and spin-orbit coupling. We reported the electronic density states calculations and the related optical properties as well as the Fermi surface. The near-Fermi valence bands are mainly composed of La-p/d states and show a substantial overlapping with the conduction band, proving a metallic nature of the perovskite. The Fermi surfaces, comprises of the disconnected electronic-like pockets and hole-like pockets situated around Z. The optical properties were also discussed to complete the optoelectronic structure description.
\end{abstract}

(c) 2019 Elsevier B.V. All rights reserved.

\section{Introduction}

One of the main entities of natural gas is methane, available as abundant raw material. Natural gas is source of driving fuels for most of the vehicles. It has a low emission during cold start and low particulate matter as compared to petroleum. It emits less $\mathrm{CO}_{2}$ due to low carbon to hydrogen ratio hence; contributes less to greenhouse effect. After all exhaust stream of natural CO gas that unburned methane powerful constituents of greenhouse gas. Vehicles operating on natural gas under stoichiometric fuel to air condition also emit $\mathrm{NO}_{\mathrm{x}}$ but lean-burn natural gas fueled vehicles (NGFVs) that operate at low temperature $(<550)$ and does not allow $\mathrm{NO}_{\mathrm{x}}$ formation [1,2]. Therefore, stoichiometrically operating NGFVs requires three-way exhaust after-treatment for the reduction of $\mathrm{CO}$, $\mathrm{CH}_{4}$ and $\mathrm{NO}_{\mathrm{x}}$ emission. Some of the compounds of ternary oxides are potential candidates for application in the field of catalysis and electrochemistry. One such compound is $\mathrm{La}_{4} \mathrm{PdO}_{7}$, which in slightly

\footnotetext{
* Corresponding author.

E-mail address: sosaid@alfaisal.edu (S. Goumri-Said).
}

reducing atmosphere with three-way catalyst for simultaneous conversion of natural gases $\mathrm{CO}, \mathrm{C}_{3} \mathrm{H}_{6}$ and $\mathrm{NO}$ to $\mathrm{H}_{2} \mathrm{O}, \mathrm{CO}_{2}$ and $\mathrm{N}_{2}$ [3]. Catalytic behavior can be understandable by gaining information about its thermodynamic stability. Interaction between Pt-group metal and ceramic systems containing rare-earth elements with different environment can be easily understand by gaining knowledge about ternary oxides. Furthermore, thermodynamic data are very helpful in designing processes that recover rare earth and precious metal from scrap. Oxidized form of palladium (PdO) is considered to be very active catalyst for methane oxidation that has been the subject of extensive research [4-7]. Perovskite-type oxides due to low cost and high thermal stability towards high temperature sintering have been studied as catalyst for complete oxidation of hydrocarbons [8-10]. Recent works [11-15] have realized that catalysts made of noble metal-containing perovskite are pertinent for $\mathrm{CO}$ oxidation, methane oxidation to syngas, $\mathrm{CO}+\mathrm{NO}$ reaction and oxidation of volatile organic compounds. Furthermore, doped perovskite with noble metal has been found to act as fine catalyst for catalytic flameless combustion of methane [16-19]. Also it can replace efficiently three-way catalyst for gasoline and NGFVs [20-24]. 
However, judging the new performance of perovskites as threeway catalysts for NGFVs is based on operating conditions such as synthesis/preparation method, thermal treatment and active phase composition. McDaniel et al. [25] investigated stable ternary compounds, such as $\mathrm{La}_{4} \mathrm{PdO}_{7}$ and $\mathrm{La}_{2} \mathrm{Pd}_{2} \mathrm{O}_{5}$, in $\mathrm{La}-\mathrm{Pd}-\mathrm{O}$ system. However, few studies were reported on $\mathrm{LaPdO}_{3}$ and none at high pressures upon measurements have been performed on ternary compound $\mathrm{LaPdO}_{3}$ at high temperatures [26,27].

The purpose of the present research study is to offer a quantitative evaluation of the electronic structures and optical properties of $\mathrm{LaPdO}_{3}$ within computations carried out in the framework of the density functional theory (DFT). We reported a first-principles study of electronic structures, elastic and optical properties of $\mathrm{LaPdO}_{3}$ by means of full potential linearized augmented plane wave method with local the gradient approximation exchange-correlation potential and spin-orbit coupling and Hubbard corrections due to the existence of Pd and La elements respectively.

\section{Calculation methodology and crystal structure description}

Our calculations were carried out using the full potential linearized augmented plane wave (FP-LAPW) [28] as implemented in WIEN2k code [29-31]. We used the exchange-correlation potential within the local density approximation (LDA) [32], generalized gradient approximation (GGA) [33] and Engel and Vosko's generalized gradient approximation (EV-GGA) [34]. It is recognized that both LDA and GGA approaches have demonstrated to be not sufficiently flexible to accurately reproduce both the exchangecorrelation energy and its charge derivative. So the Engel and Vosko (EV-GGA) deliberate on this short coming and put up the new functional form of GGA, which is capable to better reproduce the exchange potential. Furthermore, we added Hubbard correction, $\mathrm{U}=5 \mathrm{eV}$, to treat La-f orbitals [35]. We have considered the relativistic spin-orbit with second variation formalism [35-37]. In the FP-LAPW method, the space is categorized into nonoverlapping muffin-tin (MT) spheres unconnected by an interstitial region. The non-overlapping atomic spheres region is used as a linear combination of radial functions times spherical harmonics, whereas the wave expansion is used in the interstitial region a plane. In this calculation, the $\mathrm{R}_{\mathrm{mt}} \times \mathrm{K}_{\mathrm{max}}=7\left(\mathrm{R}_{\mathrm{mt}}\right.$ is known as the smallest of all MT sphere radius and $K_{\max }$ is the maximum value of the wave vector $\mathrm{K}$ ), while the expansion of the wave functions was set to $\mathrm{l}_{\max }=10$ inside of MTs. The sphere radii values are 2.39, 2.08 and 1.79 Bohr for La, Pd and O, respectively. The integration of the Brillouin zone is accomplished by $11 \times 10 \times 8 \mathrm{k}$-points mesh. The unit cell structure for the $\mathrm{LaPdO}_{3}$ compound is displayed in Fig. 1. It shows an orthorhombic symmetry with space group, Pbnm (\# 62), with lattice constant $\mathrm{a}=5.5898 \AA \mathrm{A}, \mathrm{b}=5.8502 \AA$, and $\mathrm{c}=7.8665 \AA$ $[25,26]$. All details of the relaxation and optimization process were reported in the supplementary information.

\section{Results and discussion}

\subsection{Electronic structure, bonding characterization and fermi surface}

Band structures and densities of states (DOS), were computed at the equilibrium geometry, as illustrated in Figs. 2 and 3. We portrayed the near-Fermi band structure along the selected highsymmetry points inside the first Brillouin zone (BZ) of the bodycentered orthorhombic structure. The Fermi level $\left(E_{F}\right)$ is set to valence band maximum. From the electronic band structure, a partially occupied band overlaps the Fermi level in the vicinity of Y$\mathrm{R}$ point, leading to the metallic nature of $\mathrm{LaPdO}_{3}$. As shown in Fig. 3, the attained value of the total density of states at the $\mathrm{E}_{\mathrm{F}}$ is 0.82

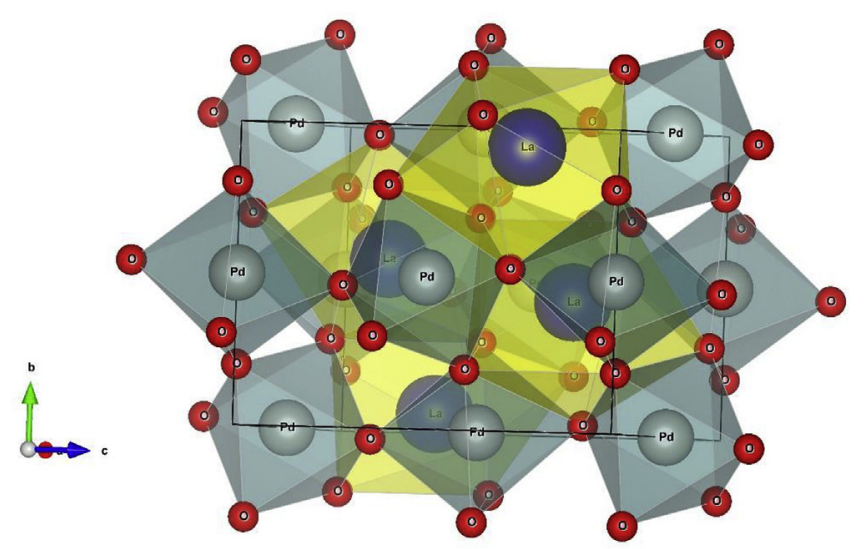

Fig. 1. Crystal structure and unit cell for DFT calculations.

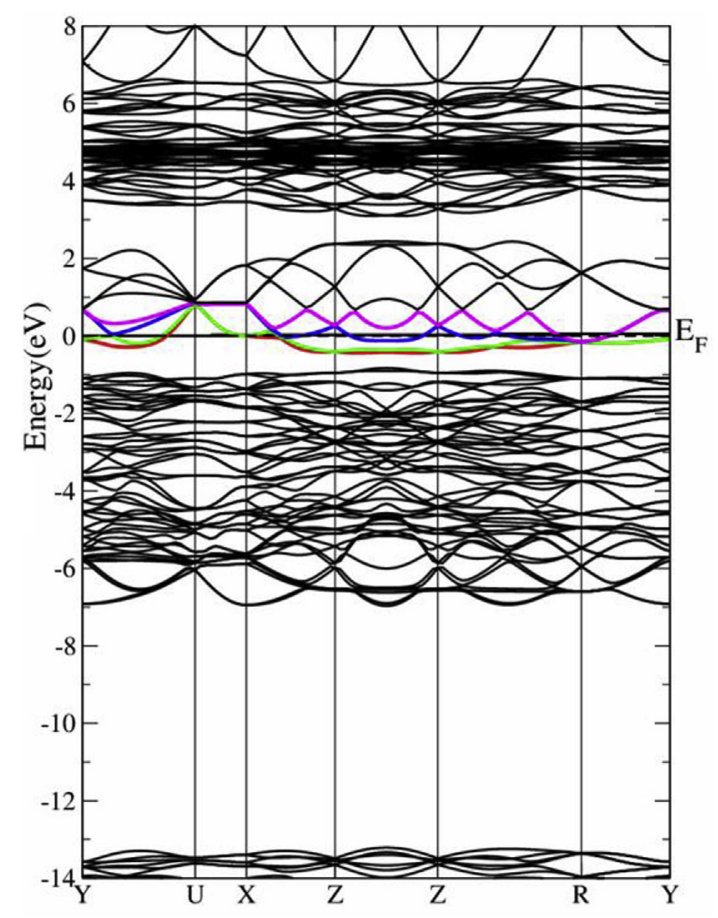

Fig. 2. Calculated electronic band structures for understanding the electronic band gap nature and contributing bands.

electrons/eV. The partial densities of states (LDOS) are displayed in Fig. 3 to indicate the hybridization between the electronic states of elements La, Pd and O. It indicates that the PDOS near the $\mathrm{E}_{\mathrm{F}}$ for $\mathrm{LaPdO}_{3}$ is dominated by the localized La- $4 p / 4 d$ states. The valence band is featured by $\mathrm{O} 2 s$ and $\mathrm{Pd} 4 d$ states centered lower valence band at -15.0 to $-13.0 \mathrm{eV}$ and $\mathrm{La} 4 p / 4 d$ states lying in $\mathrm{E}_{\mathrm{F}}$. Also the low intensity states from the valence band crosses over the $E_{F}$ within the conduction band. It is clearer in Fig. 3, that a narrower yaxis range show the La $p / d / f$, Pd $d$ and $O$ atom projected PDOS four different structure sites. $\mathrm{O}$ atom states also contribute but with a small differences between them in addition with La and Pd, on one side and between La and Pd, on the other side. These comments concede a groundwork chemical bonding proposition entailing metals $p, d$ states with $\mathrm{O} s, p$. It is obvious that $4 p$ and $4 d$ states of Pd do not chip in significant manner at the Fermi level. The lowest valence bands from -15 to $-13 \mathrm{eV}$ below Fermi level $\left(\mathrm{E}_{\mathrm{F}}=0 \mathrm{eV}\right)$ mainly arise from $\mathrm{Pd}-4 d$ and $\mathrm{O}-2 \mathrm{~s}$ states. The higher valence bands 

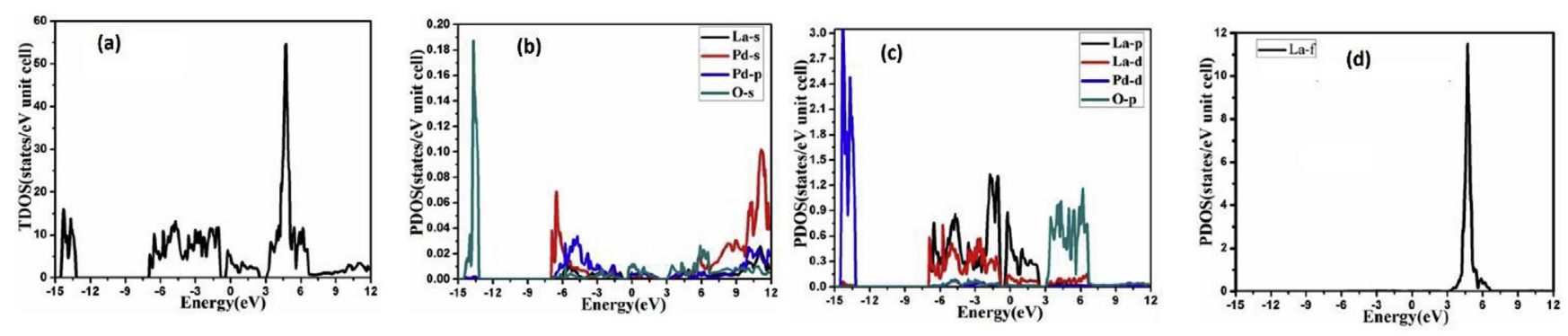

Fig. 3. Calculated total density of states (TDOS) and partial density of states (PDOS). Fermi level is considered at $0.0 \mathrm{eV}$.

between -7 and $0.0 \mathrm{eV}$ are dominated by hybridized orbitals of La $4 p / 4 d$ and $\mathrm{Pd} 4 s / 4 p$. It is obvious that the hybridization peak of Pd $4 s / 4 p$ states lies lower in energy compared to La $4 p / 4 d$ states. This suggests that the La $4 p / 4 d$ bond is become stronger than the La $4 p /$ $4 d$ bond. Furthermore, the conduction band (CB) starting from Fermi level to $12.0 \mathrm{eV}$, is mainly composed from La- $p / d / f$ states along with the small contributions from $\mathrm{O}-\mathrm{p}$ and $\mathrm{Pd}-s$ states.

To analyze the bonding character for each atom in $\mathrm{LaPdO}_{3}$, we calculated the electronic charge density distribution isosurface (see Fig. 4). The isosurface map shows tendency of ionic/covalent character within O-Pd bonds which depends on Pauling electronegativity difference of the atoms, 1.2, 2.2 and 3.5 for La, Pd and $\mathrm{O}$ atoms. It is found that the majority of charges were accumulated around $\mathrm{O}$-atom. La atom has highest electro-negativity that govern the ionic trend. Furthermore, the bond lengths and angles were also calculated, which has been listed in Table 1 . The calculated bond length and angle values show a good agreement with the experimental results [26].

Fermi surface is known as a surface that separates occupied from unoccupied electron states at zero temperature in reciprocal space. It is important to derive the shape of Fermi surface from the periodicity and symmetry of the crystalline lattice. The shape also depends on the electronic energy bands occupation. As a consequence of Pauli Exclusion Principle, the Fermi surface (FS) plots of $\mathrm{LaPdO}_{3}$ are presented in Fig. 5. In this case, we embellished the near Fermi band structure alongside the selected high-symmetry in the first Brillion zone of the orthorhombic crystal. We displayed the FS related to bands $93,94,113,114$ and 115 . In fact, it is important to consider that the site-projected l-decomposed DOSs at the Fermi level, $\mathrm{N}\left(\mathrm{E}_{\mathrm{F}}\right)$, signify that comparable endowment to $\mathrm{N}\left(\mathrm{E}_{\mathrm{F}}\right)$ comes both from La $4 p / 4 d$ states $(0.90 / 0.15$ states/eV-atom). In this way,
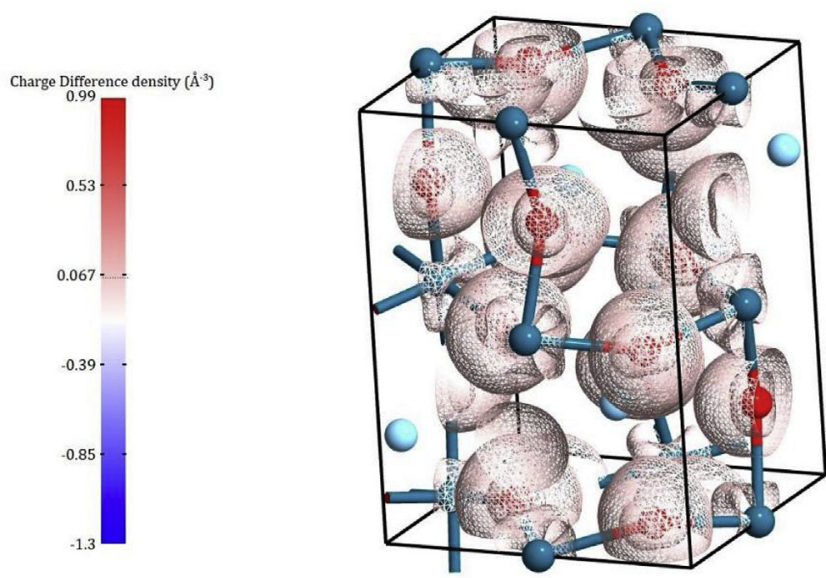

Fig. 4. Electronic charge difference density iso-surafces (3D plots). the $\mathrm{LaPdO}_{3}$ possesses quite a unique situation and might be viewed as $d$ or $s p$ metals, respectively. The spectra for the band structure demonstrate that the Fermi level is crossed by La $4 p / 4 d$ states, resulting the electrical conductivity of this material is metallic. Near the Fermi level, the existing electrons are accountable for the conductivity. Indeed, it is easy to get knowledge of the electronic structure of any metallic material from its FS as well. The band around the Fermi level exhibited a complex mixed character in combination with the quasi-flat band accompanied by series of high-dispersive bands intersecting the Fermi level, as shown in Fig. 5. FS comprises of the disconnected electronic-like pockets as well as the hole-like pockets. The closed hole-like pockets are around $\mathrm{Z}$ point, in view of the fact that the electronic-like sheets are at the X-R points of the Brillouin zone. The shaded and empty regions in the Fermi surface are illustrated the electronic sheets and the holes. Four bands crosses the $\mathrm{E}_{\mathrm{F}}$ level in the investigated compound (see Fig. 3b). FS elucidates assorted electrons in the system, whose topology is directly related to the transport characteristics of materials, such as electrical conductivity.

\section{2. optical properties}

Dielectric function $\varepsilon(\omega)$ of the electron gas depend on the frequency and has important role as it is related to various physical properties of solids. It is defined with two real and imaginary parts [38,39]: $\varepsilon(\omega)=\varepsilon_{1}(\omega)+i_{2}(\omega)$. It is well known that the imaginary part of the complex function $\varepsilon_{2}(\omega)$ in orthorhombic symmetry structure tensor can be extracted directly from the calculated electronic density of states and transition matrix elements [40] using the following expression: $\varepsilon_{2}(\omega)=\frac{2 e^{2} \pi}{\Omega \varepsilon_{0}} \sum_{k, v, c}\left|\left\langle\psi_{k}^{c}|\widehat{u} . r| \psi_{k}^{v}\right\rangle\right|^{2} \delta\left(E_{k}^{c}-\right.$ $E_{k}^{v}-E$ )where $\omega$ stands for frequency of light, $e$ is the electronic charge. $\psi_{k}^{c}$ and $\psi_{k}^{v}$ are respectively the conduction and valence band wave functions at k. $\varepsilon_{1}(\omega)$ is real part of the dielectric function and might be deduced from its imaginary part $\varepsilon_{2}(\omega)$, via Kramers-Kronig dispersion equation as follow [41,42].

$\varepsilon_{1}(\omega)=1+\frac{2}{\pi} P \int_{0}^{\infty} \frac{\omega^{\prime} \varepsilon_{2}\left(\omega^{\prime}\right)}{\omega^{\prime 2}-\omega^{2}} d \omega^{\prime}$

Moreover, the refractive index, extinction coefficient, energyloss spectrum, and the reflectivity are derived from dielectric constants $\varepsilon_{1}(\omega)$ and $\varepsilon_{2}(\omega)$. The calculated real and imaginary part of the complex dielectric function are shown in Fig. 6 (a and b). Both $\varepsilon_{1}(0)$ (static dielectric function) and low $\varepsilon_{1}(\omega)$ (real part) contingent on the band gap as implemented in Penn model relating the inverse relation with band gap $\mathrm{E}_{\mathrm{g}}[42,43]$

$\varepsilon_{1}(0) \cong 1+\left(\hbar \omega_{p} / E_{g}\right)^{2}$ 
Table 1

Calculated bond length and angle values show a good agreement with the experimental results.

\begin{tabular}{|c|c|c|c|c|c|}
\hline \multicolumn{3}{|c|}{ Bond length } & \multicolumn{3}{|l|}{ Bond angle } \\
\hline & Exp. $^{a}$ & Opt. & & Exp. $^{a}$ & Opt. \\
\hline $\mathrm{La}-\mathrm{O} 1$ & $2.27(2)$ & 2.371 & $\mathrm{O} 1-\mathrm{Pd}-\mathrm{O} 2$ & $87.9(9)$ & 89.71 \\
\hline $\mathrm{La}-\mathrm{O} 1$ & $2.50(2)$ & 2.456 & $\mathrm{O} 1-\mathrm{Pd}-\mathrm{O} 2$ & $84.2(9)$ & 87.22 \\
\hline $\mathrm{La}-\mathrm{O} 1$ & $3.43(2)$ & 3.421 & $\mathrm{O} 2-\mathrm{Pd}-\mathrm{O} 2$ & $89.4(11)$ & 89.58 \\
\hline $\mathrm{La}-\mathrm{O} 1$ & $3.55(2)$ & 3.561 & $\mathrm{Pd}-01-\mathrm{Pd}$ & $141.9(3)$ & 144.79 \\
\hline $\mathrm{La}-\mathrm{O} 2$ & $2.50(2)$ & 2.425 & $\mathrm{Pd}-02-\mathrm{Pd}$ & $153.0(6)$ & 148.57 \\
\hline $\mathrm{La}-\mathrm{O} 2$ & $2.62(2)$ & 2.619 & $\mathrm{Pd}-01-\mathrm{Pd}$ & $141.9(3)$ & 144.79 \\
\hline $\mathrm{La}-\mathrm{O} 2$ & $2.77(2)$ & 2.778 & & & \\
\hline $\mathrm{La}-\mathrm{O} 2$ & $3.54(2)$ & 3.643 & & & \\
\hline $\mathrm{Pd}-\mathrm{O} 1$ & $2.087(7)$ & 2.063 & & & \\
\hline $\mathrm{Pd}-\mathrm{O} 2$ & $2.12(2)$ & 2.102 & & & \\
\hline
\end{tabular}

a Ref. [26].

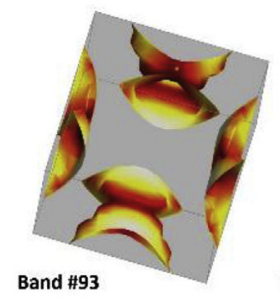

Band \#93
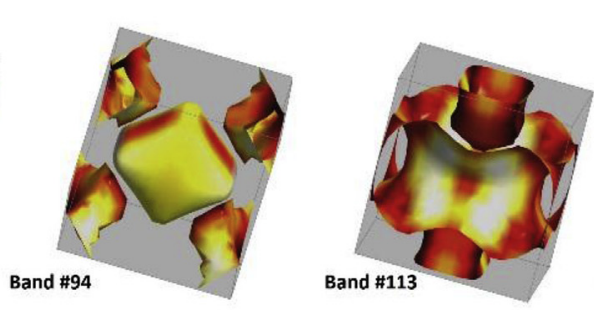
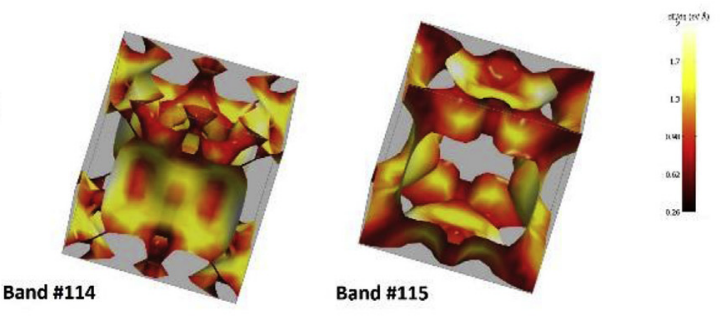

Fig. 5. Calculated Fermi surfaces iso-surafces (3D plots).

(a)

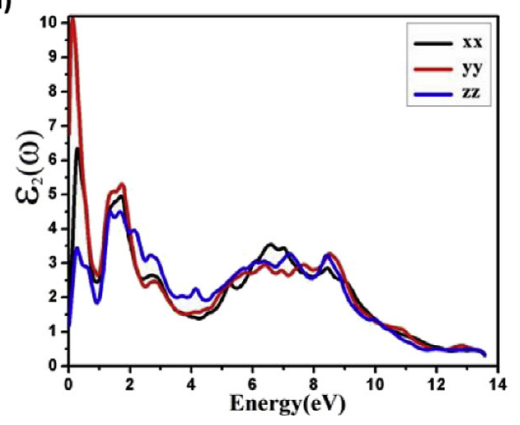

(b)

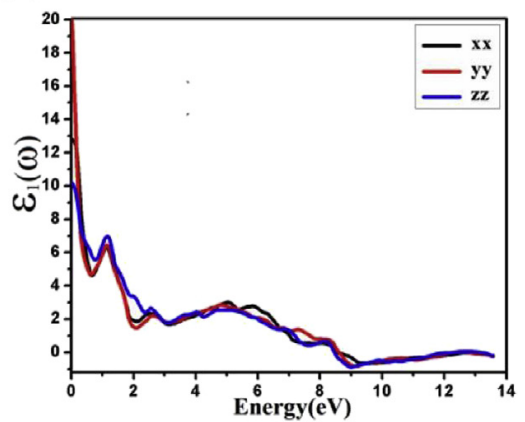

Fig. 6. Calculated imaginary (a) $\varepsilon_{2}(\omega)$ and real (b) $\varepsilon_{1}(\omega)$ parts of dielectric function.

Where $\hbar \omega_{p}$ symbolize the plasma energy. For $\mathrm{LaPdO}_{3}$, we can deduce easily from these curves that the static dielectric function values for $\varepsilon_{1}^{x x}(0)$ is $12.796, \varepsilon_{1}^{y y}(0)$ is 19.966 and $\varepsilon_{1}^{z z}(0)$ is 10.191 (arb. unit). These values are somehow considered as large because polarization dies and lattice vibrations does not contribute in the present temperature. Hence, the static $\left(\varepsilon_{1}(0)\right)$ value is associated reciprocally with the square of the energy gap $\left(\mathrm{E}_{\mathrm{g}}\right)$, therefore low and zero band gap material will possess higher value of static dielectric constant means. $\varepsilon_{1}(\omega)\left(\varepsilon_{1}^{x x}(0), \varepsilon_{1}^{y y}(0)\right.$ and $\left.\varepsilon_{1}^{z z}(0)\right)$ shows the maximum peaks at the lower energy i.e. $>2.0 \mathrm{eV}$. A sharp decrease appears in the real spectra plot, which demonstrates that this material does not interact with the photons at this range.

The spectra for absorption coefficient $I(\omega)$ is displayed in Fig. 7a. The absorption coefficient, categorizes the decay of light intensity disseminating in unit distance in medium. The computed $I(\omega)$ of $\mathrm{LaPdO}_{3}$ for three incident radiation polarizations along $I^{x x}(\omega)$ $I^{y y}(\omega)$ and $I^{z z}(\omega)(0.0-14.0 \mathrm{eV})$ are displayed in Fig. 7a. It is evident, that $I(\omega)$ spectra exhibit a noticeable anisotropy. The computed electron energy-loss function $\mathrm{L}(\omega)$ spectrum is exposed in Fig. 7c. $L(\omega)$, is a significant characteristic for identifying the energy loss of a speedy electron fleeting through a material [44].

The refractive index (real and imaginary part) $n(\omega)$ is shown in Fig. 7b. The imaginary part of refractive index shows the highest peaks at energy less than $1.0 \mathrm{eV}$, resulting the small absorption in the spectral region. The refractive index is plotted in range of $0.0-14.0 \mathrm{eV}$ (arb. unit), at $0.0 \mathrm{eV}$ the spectra show the maximum values for $n^{x x}(0), n^{y y}(0)$ and $n^{z z}(0)$, then decreases occur with increase in photon energy and almost the graph vanish at high energy, with this reason that the material absorbs high energy photons and in result the transparency lost. At $9.0 \mathrm{eV}$, the refractive index diminish lower down the unity which stipulates that the phase velocity of light is greater than the light celerity c, which look to be denial to relativity [39]. Though it seems that a signal must be transmitted as a wave packet rather than a monochromatic wave. In a dispersive medium a wave packet will propagate with a group velocity $\nu_{g}=\frac{d \omega}{d k}$ rather than the phase velocity, $\nu=\frac{\omega}{k}=\frac{c}{n^{\prime}}$ the relationship between $\nu_{g}$ and $\nu$ is given by: $\nu_{g}=\nu\left(1-\frac{k}{n} \frac{d n}{d k}\right)$

The loss function $(L(\omega))$ spectra are also calculated and displayed 


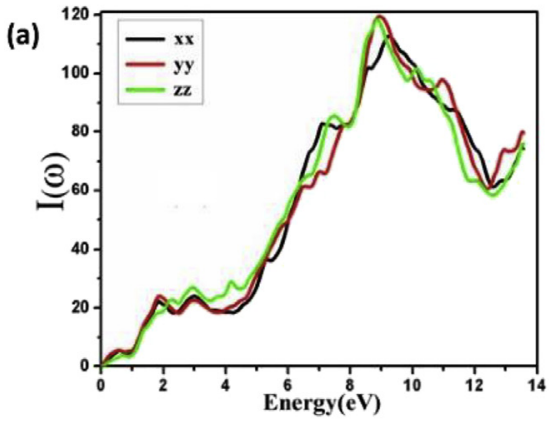

(b)

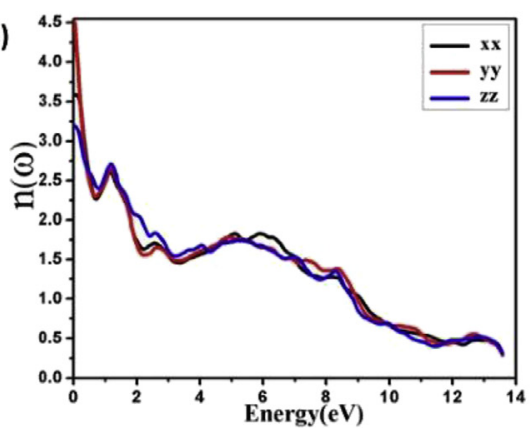

(c)

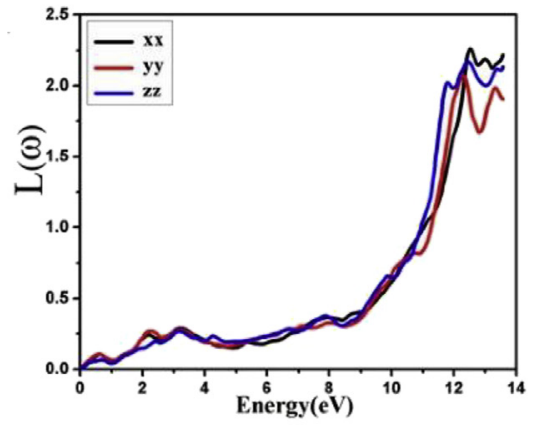

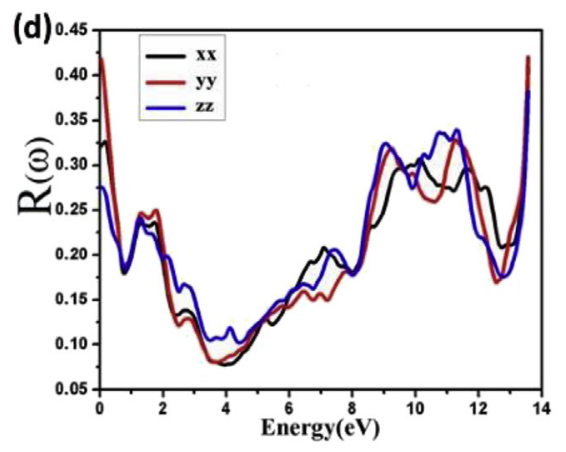

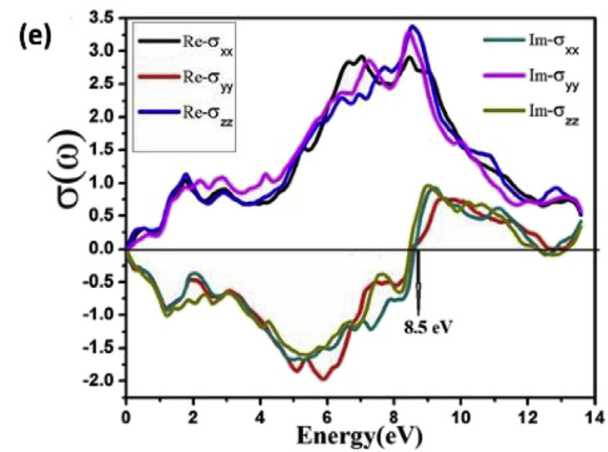

Fig. 7. (a) $I(\omega)$ Absorption spectrum, (b) refractive index $n(\omega)$, (c) energy loss $L(\omega)$, (d) reflectivity $R(\omega)$ and (e) optical conductivity (real and imaginary parts).

in Fig. 7c. The $L(\omega)$ spectra is related to the plasma frequency $\left(\omega_{p}\right)$. They exhibit a wide peak at $11.0-14.0 \mathrm{eV}$ that relay to the energy wheren $(\omega)$ and $K(\omega)$ curves cross and with the zero crossing of $\varepsilon_{1}(\omega)$. Furthermore, at $0 \mathrm{eV}$, the reflectivity $R^{x x}(0), R^{y y}(0)$ and $R^{z z}(0)$ values are estimated to $3.23,4.44$ and 2.27 (arb. unit), indicating a metallic behavior. The negative value in the $\varepsilon_{1}(\omega)$ spectra at $8.5 \mathrm{eV}$ corresponds to the peak in the reflectivity spectra. The minima of reflectivity that occurred at $4.0 \mathrm{eV}$ is corresponding to the small peak in $\varepsilon_{1}(\omega)$ and is related to the absorption of photon energy at this range (see Fig. 7a). As the energy increases with a decrease of $\varepsilon_{1}(\omega)$ occurs up to $8.5 \mathrm{eV}$ and after this energy limit, at high energy the spectra almost vanishes, which anticipate that the electromagnetic wave is mostly reflected from the medium.

The optical conductivity (real and imaginary part) is plotted in Fig. 7e, the plot consists of three main peaks, one at $2.0 \mathrm{eV}$, second at 7.0 while the prominent peak exists at $9.0 \mathrm{eV}$. These peaks correspond, the transition from $\mathrm{U}, \mathrm{R}$ and $\mathrm{Y}$ points respectively, as shown in band structures. The imaginary part of the optical conductivity spectra show an inclination with an increasing energy up to $9.0 \mathrm{eV}$. This may be caused by the deficiency in DFT (GGA). Whereas the imaginary spectra exhibit the maximum at high energies. Increasing the energy from $0.0 \mathrm{eV}$, the decrease in spectra occurs i.e. a valley is found between $2.0 \mathrm{eV}$ and $8.0 \mathrm{eV}$, giving the negative value up to $8.5 \mathrm{eV}$ after that a sharp increase and almost pass over the real part at high energy. These optical behaviors might lead us to conclude that perovskite $\mathrm{LaPdO}_{3}$ might be used for shielding from the radiation at this range beyond UV wavelengths.

\section{Conclusion}

The electronic band structures and optical properties of the perovskite $\mathrm{LaPdO}_{3}$ have been elucidated using FP-LAPW method with Hubbard model and considering the spin-orbit coupling. The study of DOS and band structures shows that the valence band (VB) along with the conduction bands $(\mathrm{CB})$ overlap substantially and many bands are crossing the Fermi level, resulting in metallic behavior. The oxygen atom states have been found to contribute in the metallic behavior but with a small differences compared to La ( $p$ and $d$ orbitals) which are the main contributors in the overlapping with Pd/O states at the Fermi level. Fermi surface (FS) topology show a 3D-like two-sheet shape made of disconnected electronic and hole-like pockets. The static dielectric function revealed the possibility of contribution from the lattice vibrations with no polarization of the dipoles.

\section{Acknowledgements}

S. Goumri-Said acknowledges financial support for this study in the form of an internal grant IRG 18418 from office of research at Alfaisal University. S. A. Khan was supported by the European Regional Development Fund, project CEDAMNF, reg. no. CZ.02.1.01/ 0.0/0.0/15_003/0000358. and GACR (Proj. 17-1484OS), CENTEM PLUS (LO1402) and CZ LD15147 of the Ministry of Education, Youth and Sports of Czechia.

\section{Appendix A. Supplementary data}

Supplementary data to this article can be found online at https://doi.org/10.1016/j.cocom.2019.e00396.

\section{References}

[1] C.S. Weaver, SAE Tech. Pap. Ser. 892133 (1989).

[2] R.J. Farrauto, R.M. Heck, Catal. Today 51 (1999) 351.

[3] M. Andersson, K. Jansson, M. Nygren, Catal. Lett. 39 (1996) 253.

[4] J.K. Lampert, M. Shahjahan Kazi, R.J. Farrauto, Appl. Catal. B Environ. 14 (1997) 211.

[5] R.M. Heck, R.J. Farrauto, Appl. Catal. Gen. 221 (2001) 443.

[6] F. Klingstedt, A. Kalandar Neyestanaki, R. Byggningsbacka, L.-E. Lindfors, M. Lunden, M. Petersson, P. Tengstrom, T. Ollonqvist, J. Vayrynen, Appl. Catal. Gen. 209 (2001) 301.

[7] P. Gelin, M. Primet, Appl. Catal. B Environ. 39 (2002) 1.

[8] T. Seiyama, L.G. Tejuka, in: J.L.G. Fierro (Ed.), Properties and Applications of Perovskite-type Oxides, Marcel Dekker, 1993, p. 215.

[9] N.H. Hong, M.B. Kanoun, J.-G. Kim, T. Sh Atabaev, K. Konishi, Satoshi Noguchi, 
Makio Kurisu, Goumri-Said Souraya, J. Phys. Chem. C 122 (2018) 2983-2989.

[10] E. Campagnoli, A. Tavares, L. Fabbrini, I. Rossetti, YuA. Dubitsky, A. Zaopo, L. Forni, Appl. Catal. B Environ. 55 (2005) 133.

[11] S. Petrovic, V. Rakic, D.M. Jovanovic, A.T. Baricevic, Appl. Catal. B Environ. 66 (2006) 249.

[12] U.G. Singh, J. Li, J.W. Bennett, A.M. Rappe, R. Seshadri, S.L. Scott, J. Catal. 249 (2007) 349.

[13] R. Zhang, H. Alamdari, S. Kaliaguine, J. Catal. 242 (2006) 241.

[14] J.-M. Giraudon, A. Elhachimi, F. Wyrwalsky, S. Siffert, A. Aboukais, J.F. Lamonier, G. Leclercq, Appl. Catal. B Environ. 75 (2007) 157.

[15] S. Cimino, G. Landi, L. Lisi, G. Russo, Catal. Today 117 (2006) 454

[16] V.R. Choudhary, B.S. Uphade, S.G. Pataskar, Fuel 78 (1999) 919.

[17] F. Cifa, P. Dinka, P. Viparelli, S. Lancione, G. Benedetti, P.L. Villa, M. Viviani, P. Nanni, Appl. Catal. B Environ. 46 (2003) 463.

[18] S. Petrovic, L. Karanovic, P.K. Stefanov, M. Zdujic, A. Terlecki-Baricevic, Appl. Catal. B Environ. 58 (2005) 133.

[19] A. Civera, G. Negro, S. Specchia, G. Saracco, V. Specchia, Catal. Today 100 (2005) 275.

[20] N. Guilhaume, M. Primet, J. Catal. 165 (1997) 197.

[21] K. Zhou, H. Chen, Q. Tian, Z. Hao, D. Shen, X. Xu, J. Mol. Catal. A Chem. 189 (2002) 225.

[22] M. Uenishi, M. Taniguchi, H. Tanaka, M. Kimura, Y. Nishihata, J. Mizuki, T. Kobayashi, Appl. Catal. B Environ. 57 (2005) 267.

[23] R. Zhang, H. Alamdari, S. Kaliaguine, Appl. Catal. B Environ. 72 (2007) 331.

[24] D. Fino, N. Russo, G. Saracco, V. Specchia, Prog. Solid State Chem. 35 (2007) 501.

[25] C.L. McDaniel, S.J. Schneider, J. Res. Natl. Bur. Stand. (U.S.) 72A (1968) 27.

[26] S. Tateno, K. Hirose, N. Sata, Y. Ohishi, Structural distortion of CaSnO3 perovskite under pressure and the quenchable post-perovskite phase as a low-pressure analogue to MgSiO3, Phys. Earth Planet. In. 181 (2010) 54-59.

[27] Seung-Joo Kim, Sylvain Lemaux, Gerard Demazeau, Jong-Young Kim, JinHo Choy, J. Mater. Chem. 12 (2002) 995-1000.

[28] D. Singh, Planes Waves, Pseudo-potentials and the LAPW Method, Kluwer Academic Publishers, Boston, Dortrecht, London, 1994.

[29] P. Blaha, K. Schwarz, G.K.H. Madsen, D. Hvasnicka, J. Luitz, WIEN2k, An Augmented Plane Wave Plus Local Orbitals Program for Calculating Crystal Properties, Vienna University of Technology, Austria, 2001.

[30] K.M. Wong, S.M. Alay-e-Abbas, A. Shaukat, Y. Fang, Y. Lei, J. Appl. Phys. 113 (2013) 014304.

[31] K.M. Wong, S.M. Alay-e-Abbas, Y. Fang, A. Shaukat, Y. Lei, J. Appl. Phys. 114 (2013) 034901.

[32] J.R. Perdew, Y. Wang, Phys. Rev. B 45 (1996) 13244.

[33] J.P. Perdew, K. Burke, M. Ernzerhof, Phys. Rev. Lett. 77 (1996) 3865.

[34] E. Engel, S.H. Vosko, Phys. Rev. B 50 (1994) 10498.

35] N.E. Christensen, J. Phys. F Met. Phys. 8 (1978) L51.

[36] M.B. Kanoun, A.H. Reshak, N. Kanoun-Bouayed, S. Goumri-Said, J. Magn. Magn. Mater. 324 (2012) 1397-1405.

[37] S. Azam, S.A. Khan, J. Minar, W. Khan, H. Ud Din, R. Khenata, G. Murtaza, S. BinOmran, S. Goumri-Said, Semicond. Sci. Technol. 30 (2015) 105018.

[38] M.B. Kanoun, S. Goumri-Said, Semicond. Sci. Technol. 23 (12) (2008) 125036

[39] B. Amin, I. Ahmad, M. Maqbool, S. Goumri-Said, R. Ahmad, J. Appl. Phys. 109 (2011) 023109.

[40] S. Azam, A.H. Reshak, Mater. Sci. Semicond. Process. 26 (2014) 649-656.

[41] F. Wooten, Optical Properties of Solids, Academic Press, New York, 1972.

[42] D. Penn, Phys. Rev. B 128 (1962) 2093.

[43] S. Goumri-Said, M.B. Kanoun, J. Solid State Chem. 197 (2013) 304-311.

[44] M.B. Kanoun, S. Goumri-Said, A.H. Reshak, Comput. Mater. Sci. 47 (2009) $491-500$. 